\title{
ACOUSTIC ABSORPTION OF POROUS SURFACING WITH DUAL POROSITY
}

\author{
C. BOUTIN $\dagger$ \\ Laboratoire Géomatériaux, DGCB, CNRS URA 1652, rue Maurice Audin, \\ 69518 Vaulx-en-Velin, Cedex, France \\ P. ROYER \\ Department of Petroleum Engineering, Heriot-Watt University, Edinburgh EH14 4AS, U.K.
}

and

J. L. AURIAULT

Laboratoire "Sols, Solides, Structures", INPG, UJF, CNRS UMR 5521, BP 53X,

38041 Grenoble, Cedex, France

\begin{abstract}
This paper is devoted to the determination of the acoustic characteristics of a porous medium saturated by air. The analysis of sound propagation in such a medium is performed using an homogenization technique. This theory is suitable since acoustic wavelengths are much greater than the usual pore size. The macroscopic descriptions involve the effects of air viscosity, inertial forces and heat transfer.

The first part of the paper deals with single porosity materials. Two cases are investigated : (i) a medium with large pores in which thermal exchanges are negligible; (ii) a medium with smaller pores for which thermal exchanges must be accounted for.

The second part is concerned with dual porosity media, i.e. when the grains themselves are also porous. Neglecting heat transfer first yields a simplified macroscopic description. This simply dual porosity model is then improved by considering thermal effects.

These results show that new porous materials could be evolved by introducing a microporosity structure that would give enhanced absorption properties over a wide range of frequencies. (C) 1998 Elsevier Science Ltd. All rights reserved.
\end{abstract}

\section{INTRODUCTION}

Dry porous media, i.e. media saturated by air, present interesting acoustic properties for reducing the level of ambient noise. Inside buildings, such media are now very frequently used as wall lining. On the outside, the design of anti-noise walls, for instance, takes advantage of these properties to reduce sound intensity in the vicinity of railways or motorways. The materials used for this purpose are often coarse, made of granular aggregates and have a pore size ranging from few millimetres to few centimetres. Another example is that of porous road surfacing used in road engineering. These materials, which present a large intrinsic permeability $\left(10^{-9} \mathrm{~m}^{2}\right)$ are known for decreasing traffic noise from 20-10 dB (Bar and Delanne, 1993).

The key point about absorption is that when an acoustic wave arrives on a previous surface, air is pushed within the pores. Therefore, only a partial reflection of the wave occurs, and the transmitted wave is damped. The description of these phenomena requires the physical analysis of gas flow in the pores. The reader is referred to Allard (1993) for a detailed presentation of these aspects.

In this paper we use the four main following hypotheses : 
(i) the porous skeleton is assumed to be perfectly rigid because of the weak level of acoustic pressure,

(ii) air is considered as a viscous fluid and its flow in the pores is governed by NavierStokes equation,

(iii) air compressibility is affected by thermal exchanges with the solid, which are described by Fourier's equation,

(iv) since acoustic motions are very small, convection or advection effects are negligible, so that the non-linear terms are ignored.

In order to derive the acoustic behaviour of such a medium, we use the homogenization method developed by Sanchez-Palencia (1980). The two fundamental assumptions which have to be satisfied for applying this method are firstly, the existence of a representative elementary volume (REV) and secondly, the separation of scales, which imposes the condition that the macroscopic size - here the acoustic wavelength - must be much greater than the characteristic size of the REV.

Basically, the homogenization technique is an asymptotic method based on two spacevariables, i.e. one for each scale. The macroscopic description which corresponds to the first significant order, is an approximation of the real behaviour. An estimation of the accuracy of the homogenized behaviour is given by the scale ratio of the macroscopic characteristic length to the macroscopic characteristic length.

This approach initially developed for solving two scale problems was recently extended for solving three scale problems by Auriault and Boutin (1993-1994). In the present work, both circumstances are considered since we focus on single porosity materials in the first part (two scales) and on dual porosity media, i.e. when the solid matrix material is also porous (three scales), in the second part.

In Section 2, two models for single porosity media are presented. We first consider the case of coarse materials having large pores, so that the assumption of adiabatic perturbations in air is valid. The macroscopic description can be compared to Biot's theory. However, since the solid is rigid, the only acoustic wave is of the $P_{2}$ type (Biot, 1956). For finer materials, thermal exchanges have to be taken into account. The homogenization approach leads to the same model as that presented by Attenborough (1983). This description includes two dissipative effects, one due to the viscosity and the other due to thermal non-equilibrium, and is in close agreement with the measurements performed on porous materials, for example by Allard et al. (1993).

In Section 3, the possibility of enhancing absorption properties by considering dual porosity media is investigated. In this case the grains of the skeleton are also assumed to be porous. This configuration can easily be realised for coarse materials. The following analysis also allows to investigate the case where there is a wide range of pore-sizes.

Modelling dual porosity media has already been performed in petroleum engineering, in order to model fractured porous reservoirs. The first work on quasi-static flow through dual porosity reservoirs was developed by Barenblatt et al. (1960) using a phenomenological approach. Statics and dynamics in such media were treated using an homogenization method in Auriault and Royer (1993), Royer and Auriault (1994), Royer et al. (1996), Royer (1994), Boutin (1994), Auriault and Boutin (1993-1994). It was proved in these latter papers that the most interesting case is obtained when scale ratios between wavelength and pores, and between pores and micropores are identical. The question of dual porosity media within the context of acoustics has already been tackled in Boutin et al. (1996). To our knowledge, this was the first study on the subject. The models which are presented in Section 2 (single porosity media) are no longer valid when there is a microporous system. In effect, the physics in the micropores is different from that in the pores.

Firstly, a simple macroscopic model of dual porosity is derived by assuming adiabatic perturbations for air in the pore space, and isothermal conditions in the micropore space. The difference between this model and the single porosity model is the coupling of air flux between pores and micropores, which is related to the diffusion of the pore pressure in the micropores. This phenomenon shows memory effects that results in an additional dissipation effect at high frequencies. Then, the description is improved by taking thermal 
exchanges into account in both pores and micropores. At high frequencies, this provides another dissipation term due to the thermal non-equilibrium in the micropores. Finally, the characteristic frequencies associated with the different contributions to dissipation (viscosity, thermal exchanges, pressure diffusion) are calculated for two distinct dual porosity configurations. Two simple numerical results are presented.

\section{SINGLE POROSITY MEDIUM}

In this section we deduce the acoustic properties of a single porosity medium by using the homogenization method. For the sake of simplicity we proceed in two steps. In the first part we consider a medium with large pores, for which the adiabatic approximation can be applied. In the second part, we develop the model to include the effects of thermal exchanges. The study is conducted under harmonic regime.

These results and their derivation will be used again in Section 3 for modelling dual porosity systems. For this purpose, the homogenization procedure is presented in detail in the present section.

\subsection{Modelling sound propagation through a rigid single porosity medium}

2.1.1. Medium description. The first main assumption, which is common to all homogenization methods, is the existence of a representative elementary volume (REV). With the method of homogenization for periodic structures, it is also assumed that the medium is periodic, and therefore, that the REV is the periodic cell.

Thus, consider the medium to be $\Omega$-periodic and the period characteristic length to be $l$. The solid and the pores occupy the domains $\Omega_{s}$ and $\Omega_{p}$, respectively, and their common boundary is $\Gamma$ (Fig. 1).

$$
\phi=\frac{\left|\Omega_{p}\right|}{|\Omega|} \text { is the porosity. }\langle\cdot\rangle_{\Omega}=\frac{1}{|\Omega|} \int_{\Omega_{p}} \cdot \mathrm{d} \Omega \quad \text { denotes the average over the period }
$$

The second main assumption is the separation of scales. It means that the microscopic characteristic length $l$ must be small compared to the macroscopic size of the volume and to the wavelength. Let $L$ be the macroscopic characteristic length. Therefore, $L$ is the smallest length between the macroscopic size of the volume and the wavelength. In acoustics, $L$ can be related to the wavelength by the following equality (Boutin and Auriault, 1990):

$$
L=\frac{\lambda}{2 \pi}
$$

The separation of scales is expressed as follows:

$$
\varepsilon=\frac{1}{L} \ll 1
$$

2.1.2. Governing equations. At the pore scale, the flow of air through $\Omega_{p}$ is governed by the momentum balance equation, the mass balance equation, the air state equation and the adherence condition on the boundary $\Gamma$.

In this work, all non-linear effects (convection, advection) are neglected. This assumption is justified by the low level of pressure which, in the context of acoustics, induces very small perturbations.

To know the thermodynamical conditions of the system, the pore size must be compared to the thickness $\delta_{t}$ of the thermal layer, in which thermal exchanges may occur. Under harmonic regime at pulsation $\omega, \delta_{t}$ is defined as follows: 


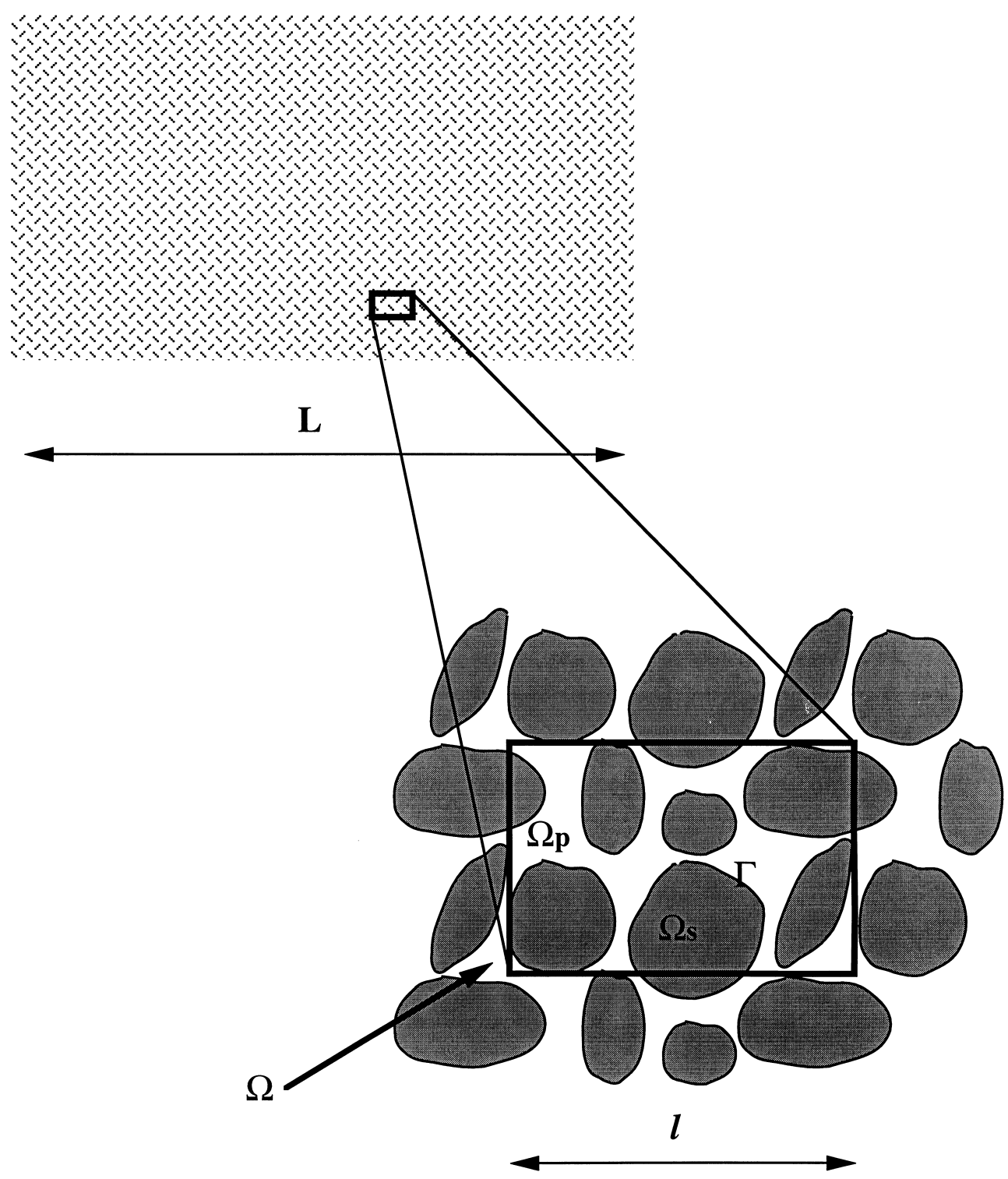

Fig. 1. Single porosity medium: description of the periodic cell at the pore scale.

$$
\delta_{t}=\sqrt{\frac{\kappa}{\rho^{e} c_{\mathrm{p}} \omega}}
$$

where $\rho^{e}$ is the density, $c_{\mathrm{p}}$ is the specific mass capacity and $\kappa$ is the thermal conductivity of the medium under consideration. Typical thermal characteristics are shown in Table 1.

Table 1. Typical thermal characteristic in air and in the grains

\begin{tabular}{lccc}
\hline & $\kappa(\mathrm{W} / \mathrm{mK})$ & $\rho\left(\mathrm{kg} / \mathrm{m}^{3}\right)$ & $C_{\mathrm{p}}(\mathrm{J} / \mathrm{Kg})$ \\
\hline Air & 0.026 & 1.23 & 1000 \\
Grains & 1.4 & 2500 & 837 \\
\hline
\end{tabular}


In the acoustic domain defined by

$$
50 \mathrm{~Hz}<f<20 \mathrm{kHz}\left(f=\frac{\omega}{2 \pi}\right)
$$

the thermal layer thickness in air and in the grains are such that:

$$
\text { Air : } 13 \mu \mathrm{m}<\delta_{t}<0.26 \mathrm{~mm}
$$

Grains : $\quad 2.3 \mu \mathrm{m}<\delta_{t}<46 \mu \mathrm{m}$

In this section we assume the medium to be made of large pores, as it is typically the case in porus road surfacing. For a characteristic pore size $l \approx 1 \mathrm{~mm}$, the condition $\delta_{t}<l$ is checked for each constituent and thus, thermal exchanges can be neglected. As a result, air can be considered as being in adiabatic conditions. Hence, we have the following relationship between pressure and density variations:

$$
\frac{P}{P^{e}}=\gamma \frac{\rho}{\rho^{e}}
$$

where $\gamma$ is the specific heat ratio, $P^{e}$ and $\rho^{e}$ are the pressure and the density at the equilibrium, and $P$ and $\rho$ are pressure and density variations, respectively.

Thus the governing equations are the following:

Momentum balance (linearized Navier-Stokes equation) in $\Omega_{p}$

$$
\mu \Delta \mathbf{v}+(\lambda+\mu) \nabla(\nabla \cdot \mathbf{v})-\nabla P=\rho^{e} \frac{\partial \mathbf{v}}{\partial t}
$$

where $v$ is the velocity, and $\mu$ and $\lambda$ are the shear and the volume viscosities of air, respectively.

Mass balance (continuity equation) in $\Omega_{p}$

$$
\frac{\mathrm{d} \rho}{\mathrm{d} t}+\rho^{e} \boldsymbol{\nabla} \cdot \mathbf{v}=0
$$

Air state equation (adiabatic)

$$
P=\frac{P^{e}}{\rho^{e}} \gamma \rho
$$

Adherence condition on $\Gamma$

$$
\mathbf{v} / \Gamma=\mathbf{0}
$$

2.1.3. Dimensionless numbers. The objective is to express the governing equations in a dimensionless form. For this purpose, we may estimate the dimensionless numbers introduced by both balance eqns (1), (2):

$$
Q=\frac{|\nabla P|}{|\mu \Delta \mathbf{v}|}, \quad R t=\frac{\left|\rho^{e} \frac{\partial \mathbf{v}}{\partial t}\right|}{|\mu \Delta \mathbf{v}|}, \quad S=\frac{\left|\frac{\mathrm{d} \rho}{\mathrm{d} t}\right|}{\left|\rho^{e} \mathbf{\nabla} \cdot \mathbf{v}\right|}
$$

To evaluate these numbers, we use $P$ and $V$ a characteristic values of the acoustic pressure amplitude and of the velocity, respectively. From the physical point of view, the flow is forced by the macroscopic pressure gradient. Then, we have: 


$$
|\nabla P|=O\left(\frac{P}{L}\right)
$$

In addition, since the flow occurs in the pores, the viscous forces are such that:

$$
|\mu \Delta \mathbf{v}|=O\left(\mu \frac{V}{l^{2}}\right)
$$

Since we consider harmonic perturbations at pulsation $\omega$, the order of magnitude of the inertial term is given by:

$$
\rho^{e} \frac{\partial \mathbf{v}}{\partial t}=O\left(\rho^{e} \omega V\right)
$$

Finally, since we investigate sound propagation with a wavelength $\lambda=2 \pi L$, the volume variation of air is estimated by:

$$
\left|\rho^{e}(\boldsymbol{\nabla} \cdot \mathbf{v})\right|=O\left(\frac{\rho^{e} V}{L}\right)
$$

Let us consider the situation of greatest interest, which is when the three forces in NavierStokes equation are of the same order of magnitude. In other words, the pressure gradient is balanced by both viscous and inertial terms.

$$
\rho^{e} \omega V=O\left(\frac{P}{L}\right)=O\left(\mu \frac{V}{l^{2}}\right)
$$

From this we deduce:

$$
\frac{\frac{P}{L}}{\mu \frac{V}{l^{2}}}=O(1), \quad \rho^{e} \frac{\partial \mathbf{v}}{\partial t}=O\left(\frac{P}{L}\right)
$$

Let us note that this latter assumption means that the thickness of the viscous skin: $\delta_{v}=\sqrt{\mu / \rho^{e} \omega}$ is of the order of the pore size. This is in good agreement with reality since the kinematic air viscosity is :

$$
\frac{\mu}{\rho^{e}}=1.5 \times 10^{-5} \mathrm{~m}^{2} / \mathrm{s}
$$

which, in the acoustic range of frequencies $(50 \mathrm{~Hz}-20 \mathrm{kHz})$, gives :

$$
10 \mu \mathrm{m}<\delta_{v}<2.2 \mathrm{~mm}
$$

As for $S$, since spatial volume variations are balanced by time density variations, we have:

$$
\left|\rho^{e}(\boldsymbol{\nabla} \cdot \mathbf{v})\right|=O\left(\frac{\rho^{e} V}{L}\right)=O(\omega \rho)
$$

Now, we choose the macroscopic characteristic length $L$ as reference length. The values of 
$Q, R t$ and $S$ are then designated by $Q_{L}, R t_{L}$ and $S_{L}$, respectively, and their expressions are the following:

$$
Q_{L}=\frac{\frac{P}{L}}{\mu \frac{V}{L^{2}}}, \quad R t_{L}=O\left(\frac{\rho^{e} \omega L^{2}}{\mu}\right), \quad S_{L}=O\left(\frac{\omega \rho}{\frac{\rho^{e} V}{L}}\right)
$$

Therefore, considering the above physical analysis, we get:

$$
\begin{aligned}
Q_{L} & =O\left(\varepsilon^{-2}\right) \\
R t_{L} & =O\left(\varepsilon^{-2}\right) \\
S_{L} & =O(1)
\end{aligned}
$$

Thus, when cast in dimensionless form with $L$ as reference length, the governing equations for harmonic pulsations are written as follows (the term $e^{i \omega t}$ is omitted) :

$$
\begin{gathered}
\varepsilon^{2} \mu \Delta \mathbf{v}+\varepsilon^{2}(\lambda+\mu) \nabla(\boldsymbol{\nabla} \cdot \mathbf{v})-\nabla P=i \omega \rho^{e} \mathbf{v} \\
i \omega \rho+\rho^{e} \boldsymbol{\nabla} \cdot \mathbf{v}=0 \\
P=\frac{P^{e}}{\rho^{e}} \gamma \rho \\
\mathbf{v} / \Gamma=\mathbf{0}
\end{gathered}
$$

Notice that all quantities are now dimensionless quantities, but for the sake of simplicity, we keep the same notations.

As a result of the separation of scales, two independent dimensionless space variables can be defined. Let $\mathbf{X}$ be the physical space variable of the system. We define :

$\bullet \mathbf{y}=\mathbf{X} / l$ as being the microscopic dimensionless space variable

$\bullet \mathbf{x}=\mathbf{X} / L$ as being the macroscopic dimensionless space variable

Pressure, velocity and density fields are, a priori, functions of both variables.

The gradient operator $\boldsymbol{\nabla}$ is a scaled and dimensionless quantity as well. It may now be written as follows:

$$
\boldsymbol{\nabla}_{x}+\varepsilon^{-1} \nabla_{y}
$$

2.1.4. Homogenization. The upscaling process may now be performed. The variations of pressure, velocity and density amplitudes are looked for in the form of asymptotic expansions in power of $\varepsilon$ :

$$
\begin{aligned}
& P(\mathbf{x}, \mathbf{y})=\varepsilon^{0} P^{0}(\mathbf{x}, \mathbf{y})+\varepsilon^{1} P^{1}(\mathbf{x}, \mathbf{y})+\varepsilon^{2} P^{2}(\mathbf{x}, \mathbf{y})+\cdots \\
& \mathbf{v}(\mathbf{x}, \mathbf{y})=\varepsilon^{0} \mathbf{v}^{0}(\mathbf{x}, \mathbf{y})+\varepsilon^{1} \mathbf{v}^{1}(\mathbf{x}, \mathbf{y})+\varepsilon^{2} \mathbf{v}^{2}(\mathbf{x}, \mathbf{y})+\cdots \\
& \rho(\mathbf{x}, \mathbf{y})=\varepsilon^{0} \rho^{0}(\mathbf{x}, \mathbf{y})+\varepsilon^{1} \rho^{1}(\mathbf{x}, \mathbf{y})+\varepsilon^{2} \rho^{2}(\mathbf{x}, \mathbf{y})+\cdots
\end{aligned}
$$

where $P^{i}, \mathbf{v}^{i}$ and $\rho^{i}$ are $\Omega$-periodic, with respect to the variable $\mathbf{y}$.

These expansions must now be introduced in the dimensionless set of eqns (5)-(8). Then the boundary-value problems at the successive orders of $\varepsilon$ (defined over the periodic cell), must be resolved so as to derive the macroscopic description. 
From eqn (5), we get at the first two orders $O\left(\varepsilon^{-1}\right)$ and $O\left(\varepsilon^{0}\right)$ :

$$
\begin{gathered}
\nabla_{y} P^{0}=\mathbf{0} \\
\mu \Delta_{y} \mathbf{v}^{0}+(\lambda+\mu) \nabla_{y}\left(\nabla_{y} \cdot \mathbf{v}^{0}\right)-\nabla_{y} P^{1}-\nabla_{x} P^{0}=i \omega \rho^{e} \mathbf{v}^{0}
\end{gathered}
$$

Similarly, we get from (6) :

$$
\begin{gathered}
\nabla_{y} \cdot \mathbf{v}^{0}=0 \\
i \omega \rho^{0}+\rho^{e} \nabla_{x} \cdot \mathbf{v}^{0}+\rho^{e} \nabla_{y} \cdot \mathbf{v}^{1}=0
\end{gathered}
$$

At the first order, eqn (7) gives:

$$
P^{0}=\frac{P^{e}}{\rho^{e}} \gamma \rho^{0}
$$

Finally, the adherence condition (8) gives:

$$
\mathbf{v}^{0} / \Gamma=\mathbf{v}^{1} / \Gamma=\cdots=\mathbf{0}
$$

We may now proceed to solve the successive boundary-value problems.

From eqns (9) and (13) it is clear that:

$$
\begin{aligned}
P^{0} & =P^{0}(\mathbf{x}) \\
\rho^{0} & =\rho^{0}(\mathbf{x})
\end{aligned}
$$

Equations (10), (11) and (14) describe the dynamic flow of an incompressible viscous fluid in a porous cell. This specific problem involves dynamic permeability and was solved by Levy (1979) and Auriault (1980). The velocity $\mathbf{v}^{0}$ is expressed as:

$$
\mathbf{v}^{0}=-\frac{\tilde{k}}{\mu} \nabla_{x} P^{0}
$$

where $\tilde{k}$ is a complex valued tensor which depends on the local variable, $\mathbf{y}$, and also on the dimensionless pulsation $\omega / \omega_{c}$, where $\omega_{c}$ is the characteristic pulsation, of the order of $\mu / \rho^{e} l^{2}$.

Now, considering the $\Omega$-periodicity of $\mathbf{v}^{1}$ together with the adherence condition (14) and then integrating eqn (12) over the period yields :

$$
\phi i \omega \rho^{0}+\rho^{e} \boldsymbol{\nabla}_{x} \cdot\left\langle\mathbf{v}^{0}\right\rangle_{\Omega}=0
$$

where

$$
\left\langle\mathbf{v}^{0}\right\rangle_{\Omega}=-\frac{\tilde{K}}{\mu} \nabla_{x} P^{0}
$$

and

$$
\tilde{K}=\langle\tilde{k}\rangle_{\Omega}=\frac{1}{|\Omega|} \int_{\Omega_{p}} \tilde{k} \mathrm{~d} \Omega
$$

Equations (13), (18) and (19) describe acoustics of a medium made of large pores saturated 
by air. $\tilde{K}$ is the dynamic permeability tensor. Combining these three equations, the macroscopic description can be rewritten as follows:

$$
\frac{\phi i \omega}{\gamma P^{e}} P^{0}-\nabla_{x} \cdot\left(\frac{\tilde{K}}{\mu} \nabla_{x} P^{0}\right)=0
$$

2.1.5. Properties of $\tilde{\mathrm{K}}$. The acoustic features of the system are related to the properties of the dynamic permeability $\tilde{K}$. These properties are described in detail for example in Auriault et al. (1985) or in Sheng and Zhou (1988) for a medium saturated by a liquid. The main results are summarised below for the case of an isotropic medium.

At low frequencies, viscous effects are predominant and $K$ tends towards the realvalued intrinsic permeability: $K(0)=O\left(\phi l^{2}\right)$. Thus, in this case we find the classical Darcy's law. $K(0)$ is related to the flow resistivity, $\sigma$, that is commonly used in acoustics, by:

$$
\sigma=\mu / K(0) .
$$

At high frequencies, inertial effects dominate and then $K$ tends towards a pure imaginary value : $K(\infty)=\phi \mu / i \omega \rho^{e} \alpha_{\infty}$. Hence, at high frequencies, the dynamic Darcy's law tends towards a classical dynamic equation in which air density is corrected by the tortuosity $\alpha_{\infty}$, which highlights the influence of the "added" mass. Low and high frequency domains are delimited by a critical frequency value which is of the order of $O\left(\mu / \rho^{e} l^{2}\right)$. A more accurate estimate of this frequency is obtained by equalising viscous and inertial effects of the macroscopic flow:

$$
\omega_{c}=\frac{\mu \phi}{K(0) \rho \alpha_{\infty}}
$$

The dynamic permeability may be related to the effective density—which is commonly used in acoustics-by the following equality:

$$
\rho_{\mathrm{eff}}\left(\frac{\omega}{\omega_{c}}\right)=\frac{\phi \mu}{K\left(\frac{\omega}{\omega_{c}}\right)} \cdot \frac{1}{i \omega}
$$

Using dimensionless quantities, this can also be expressed in the simple form :

$$
i \omega^{*} \rho^{*} K^{*}=1
$$

where

$$
\begin{aligned}
\omega^{*} & =\frac{\omega}{\omega_{c}} \\
K^{*} & =\frac{K}{K(0)} \\
\rho^{*} & =\frac{\rho_{\mathrm{eff}}}{\alpha_{\infty} \rho^{e}}
\end{aligned}
$$

Analytical expressions can be derived for very simple duct geometries (Biot, 1956). For any pore geometry, Allard et al. (1993) have proposed an expression for $\rho^{*}$, which gives the correct asymptotic behaviour at low and high frequencies and includes the viscous layer effects : 


$$
\rho^{*}=1+\frac{\sqrt{1+i \omega^{*} / F^{2}}}{i \omega^{*}}
$$

where $F$ is a shape ratio ranging from $\sqrt{2}$ (for cylindrical pores) to 4 , with respect to the pore geometry (Sheng and Zhou, 1988).

2.1.6. Wave propagation. The complex valued acoustic velocity $C(\omega)$ may directly be deduced from eqn (20). In the isotropic case we get:

$$
C^{2}=-\frac{\gamma P^{e} K \omega}{\phi i \mu}=\frac{C a^{2} i K^{*}}{\alpha_{\infty}} \frac{\omega}{\omega_{c}}
$$

where $C a$ is the sound velocity and is defined by:

$$
C a=\sqrt{\frac{\gamma P^{e}}{\rho^{e}}}
$$

At low frequencies, we get:

$$
C(\omega) \approx C a \sqrt{\frac{i \omega^{*}}{\alpha_{\infty}}}
$$

which shows that the waves are diffusive. Then, the attenuation per wavelength tends towards one, whereas the rate of damping per metre increases with respect to $\sqrt{\omega^{*}}$.

At high frequencies, the acoustic celerity can be approached by:

$$
C(\omega) \approx \frac{C a}{\sqrt{\alpha_{\infty}}}\left[1-\frac{1}{2 F} \sqrt{\frac{1}{i \omega^{*}}}\right]
$$

Therefore, in this case the waves are propagative and attenuated. The tortuosity reduces the celerity. The attenuation per wavelength decreases with respect to $\sqrt{\omega^{*}}$, whereas the rate of damping per metre increases with respect to $\sqrt{\omega^{*}}$.

\subsection{Adding thermal effects}

The above adiabatic analysis is valid for large pores. For a more general description, and in particular for smaller pores, say $l \leqslant 0.5 \mathrm{~mm}$, thermal effects must be accounted for.

2.2.1. Heat transfer equations. We may incorporate the equation of heat conduction together with the air state equation:

$$
\begin{gathered}
\nabla \cdot(\kappa \nabla T)=i \omega\left(\rho^{e} c_{\mathrm{p}} T-P\right) \\
P=P^{e}\left(\frac{\rho}{\rho^{e}}+\frac{T}{T^{e}}\right)
\end{gathered}
$$

where $T^{e}$ is the equilibrium temperature.

In order to express the boundary conditions at the solid-air interface, let us preliminary estimate $T_{s}$, the order of magnitude of the temperature variation in the skeleton. On $\Gamma$, the temperature gradient is of the order of $T_{s} / \delta_{t s}$ and $T / \delta_{t g}$ in the solid and in air, respectively. Thus, the flux continuity sets that: 


$$
\frac{\kappa_{s} T_{s}}{\delta_{t s}}=O\left(\frac{\kappa T}{\delta_{t g}}\right)
$$

from which we deduce :

$$
T_{s}=\left(\frac{\delta_{t s} \kappa}{\delta_{t g} \kappa_{s}}\right) O(T)=\left(\sqrt{\frac{\rho^{e} c_{\mathrm{p}} \kappa}{\rho_{s} c_{\mathrm{p} s} \kappa_{s}}}\right) O(T)
$$

The air thermal impedance is much smaller than the solid thermal impedance (Table 1). As a result, $T_{s}$ is very small compared to $T$. Therefore, it will be assumed hereafter that the skeleton remains under isothermal conditions and that thermal effects are significant only in the pores. As a consequence, the thermal boundary conditions may be expressed as:

$$
T / \Gamma=0
$$

Now, eqns (24) and (25) may be scaled. The relative variations of pressure, temperature and density are of the same order of magnitude. Therefore we have:

$$
O\left(\frac{\rho}{\rho^{e}}\right)=O\left(\frac{T}{T^{e}}\right)=O\left(\frac{P}{P^{e}}\right)
$$

and, as a consequence,

$$
O\left(\rho^{e} c_{\mathrm{p}} T\right)=O(P)
$$

Thus, the only dimensionless number to be estimated is:

$$
N=\frac{\left|i \omega \rho^{e} c_{\mathrm{p}} T\right|}{|\boldsymbol{\nabla} \cdot(\kappa \nabla T)|}
$$

Since thermal exchange are assumed to occur at the pore scale, conduction and transient terms in eqn (24) are of the same order of magnitude, which expresses the fact that the thickness of the thermal layer is of the order of the pore size :

$$
\omega \rho^{e} c_{\mathrm{p}} T=O\left(\kappa \frac{T}{l^{2}}\right)
$$

As above, the reference length is the macroscopic characteristic length, $L$. We get:

$$
N_{L}=O\left(\varepsilon^{-2}\right)
$$

2.2.2. Macroscopic behaviour. As in 2.1, the set of local dimensionless equations comprises eqns (5), (6) and (8). The adiabatic state eqn (7) is replaced by eqn (25), and finally, eqns (24) and (26) must be added to the set to account for thermal effects. There is an additional unknown, $T$, which, like the other unknowns is looked for in the form of an asymptotic expansion in power of $\varepsilon$ :

$$
T(\mathbf{x}, \mathbf{y})=\varepsilon^{0} T^{0}(\mathbf{x}, \mathbf{y})+\varepsilon^{1} T^{1}(\mathbf{x}, \mathbf{y})+\varepsilon^{2} T^{2}(\mathbf{x}, \mathbf{y})+\cdots
$$

From eqns (24) and (26) at the orders of $O\left(\varepsilon^{-2}\right)$ and $O(1)$, respectively, the following problem arises : 


$$
\begin{gathered}
\nabla_{y} \cdot\left(\frac{\kappa}{i \omega \rho^{e} c_{\mathrm{p}}} \nabla_{y} \frac{T^{0}}{T^{e}}\right)-\frac{T^{0}}{T^{e}}=-\left(1-\frac{1}{\gamma}\right) \frac{P^{0}}{P^{e}} \\
T^{0} / \Gamma=0
\end{gathered}
$$

This heat transfer problem in the periodic cell has already been solved for example in Auriault (1983) and Boutin and Auriault (1993). The solution can be written in the following form :

$$
T^{0}=\frac{T^{e}}{P^{e}}\left(1-\frac{1}{\gamma}\right) g P^{0}
$$

where $g$ is a complex valued function which depends on the local variable $\mathbf{y}$ and on the dimensionless pulsation $\omega / \omega_{t}$, where $\omega_{t}$ is a characteristic thermal pulsation which is of the order of $O\left(\kappa / l^{2} \rho^{e} c_{\mathrm{p}}\right)$.

Now, considering eqn (25) at the first order gives:

$$
\frac{\rho^{0}}{\rho^{e}}=\frac{P^{0}}{P^{e}}-\frac{T^{0}}{T^{e}}=\left(1-\left(1-\frac{1}{\gamma}\right) g\right) \frac{P^{0}}{P^{e}}
$$

Equation (17), that gives the expression of $\mathbf{v}^{0}$ is still valid, but the integration of eqn (12) over the period is altered. Instead of eqn (18), we get:

$$
i \omega\left\langle\rho^{0}\right\rangle_{\Omega}+\rho^{e} \nabla_{x} \cdot\left\langle\mathbf{v}^{0}\right\rangle_{\Omega}=0
$$

where

$$
\left\langle\rho^{0}\right\rangle_{\Omega}=\phi\left[(\gamma-(\gamma-1) G] \frac{P^{0} \rho^{e}}{\gamma P^{e}}, \quad G=\frac{1}{\left|\Omega_{p}\right|} \int_{\Omega_{p}} g \mathrm{~d} \Omega\right.
$$

and

$$
\left\langle\mathbf{v}^{0}\right\rangle_{\Omega}=-\frac{\tilde{K}}{\mu} \nabla_{x} P^{0}
$$

Equations (30)-(32) give the macroscopic behaviour. Eliminating the density and the velocity, the macroscopic description can be rewritten as follows :

$$
\phi i \omega\left[1-\left(1-\frac{1}{\gamma}\right) G\right] \frac{P^{0}}{P^{e}}-\nabla_{x} \cdot\left(\frac{\tilde{K}}{\mu} \nabla_{x} P^{0}\right)=0
$$

2.2.3. Comments. Equation (33) shows that the acoustic characteristics of the system depend on the tensor $\tilde{K}$ and on the function $G$. The properties of $\tilde{K}$ remain the same as in the case of large pores (see paragraph 2.1.5). The complex valued function $G$ describes the frequency dependence of the thermal exchanges and the term $[1-(1-1 / \gamma) G] / P^{e}$ gives the macroscopic effective complex valued compressibility.

At low frequencies, transient thermal effects become negligible. Thus, considering the isothermal condition for the solid, the temperature variation tends towards zero. $G(0)=0$ and the effective compressibility tends towards the isothermal compressibility $\left(1 / P^{e}\right)$.

At high frequencies, conduction effects are negligible except in close proximity to the solid. The perturbations occur in an adiabatic way in air. $G(\infty)=1$, and the compressibility tends towards the adiabatic value $\left(1 / \gamma P^{e}\right)$. 
At medium frequencies, i.e. for pulsations of the order of $\kappa / l^{2} \rho^{e} c_{\mathrm{p}}$, there is a phase shift between temperature and pressure, and hence between density and pressure. Consequently, the effective compressibility is complex. A more accurate expression of the thermal characteristic pulsation is obtained by introducing the length $\Lambda_{t}$, defined as the ratio of the volume to the surface (Champoux and Allard, 1991):

$$
\omega_{t}=\frac{\kappa}{\Lambda_{t}^{2} \rho^{e} c_{\mathrm{p}}} \quad \Lambda_{t}=\frac{\left|\Omega_{p}\right|}{|\Gamma|}
$$

For spherical or cylindrical pores, $G$ can be expressed analytically (Attenborough, 1983; Auriault, 1983). For other geometries, the following expression gives the correct asymptotic behaviour at low and large frequencies (including the thermal layer effects) (Allard et al., 1993) :

$$
G\left(\omega_{t}^{*}\right)=\left[1+\sqrt{F_{t}^{2}+i \omega_{t}^{*}} / i \omega_{t}^{*}\right]^{-1}
$$

where $\omega_{t}^{*}$ is the dimensionless thermal frequency: $\omega_{t}^{*}=\omega / \omega_{t}$ and where $F_{t}$ is a shape ratio of the pore structure ( $F_{t}=2$ for cylindrical pores and $F_{t}=5 / 3$ for spherical pores).

The velocity of harmonic waves is determined from eqn (33). For isotropic cases we get :

$$
C^{2}=C_{a}^{2} \frac{i K^{*} \omega^{*}}{\alpha_{\infty}[\gamma-(\gamma-1) G]}
$$

Notice that the macroscopic description includes two dissipative effects, one due to the viscosity, the other one due to thermal exchanges. However, the thermal dissipation is smaller than the viscous dissipation and has a more limited range of frequencies. The thermal contribution to the rate of attenuation per wave length (i.e. to the rate of damping per metre) is directly related to the phase (i.e. to the imaginary part) of the complex valued compressibility.

From the preceding analysis, these effects are maximum for pulsations close to $\omega_{t}$. It is important to note that whereas viscous and thermal layer thicknesses are of the same order in air, $\delta_{v} / \delta_{t}=\sqrt{\mu c_{\mathrm{p}} / \kappa}=0.877$, the characteristic frequencies associated with viscous and thermal dissipation can be very different. As a matter of fact, the permeability essentially depends on the small ducts in the media, while thermal effects involve all the pores. In consequence we have the inequalities:

$$
K(0)<\Lambda_{t}^{2} \quad \text { and } \quad \omega_{t}<\omega_{c}
$$

This approach is in agreement with the phenomenological approaches of Attenborough (1983) and Allard et al. (1993). In the next section, the possibility of enhancing absorption properties by considering dual porosity media is examined.

\section{DUAL POROSITY MEDIUM}

In this section, we consider the case where the grains of the skeleton are also porous, with an open porosity $\phi^{\prime}$. In other words, $\Omega_{s}$ is a porous system. Obviously, the role of this secondary porosity depends on the size of the micropores. If the micropore size is of the order of the pore size, there is no distinction between both porous systems. Thus, considering the whole porosity $\phi+(1-\phi) \phi^{\prime}$, the description above can still be applied. Now, if the micropores are very much smaller than the pores, the grain permeability is very low. Then, the grains seem to be impervious, and the above description is valid again, considering only the largest pores. The case of interest, which is studied below, is obtained when the scale ratio of the micropore size to the pore size is identical to the scale ratio of the pore size to 
the wavelength (see for example Auriault and Royer, 1993; Royer and Auriault, 1994; Auriault and Boutin, 1993-1994).

\subsection{Modelling sound propagation through a rigid dual porosity medium}

3.1.1. Medium description. The description of the pores remains the same as before. However, since the grains are now porous, we have to describe their microstructure. Here again we consider that the microporous system has a periodic distribution. We assume that the micropores are connected to each other and to the pores. The characteristic length $l^{\prime}$ of the microporous period $\Omega^{\prime}$ is related to the pore size and to the macroscopic size by:

$$
\frac{l^{\prime}}{l}=O\left(\frac{l}{L}\right)=O(\varepsilon)
$$

At the micropore scale, the period $\Omega^{\prime}$ consists of the solid and the micropores which occupy the domains $\Omega_{m}^{\prime}$ and $\Omega_{s}^{\prime}$, respectively, and their common boundary is $\Gamma^{\prime}$ (Fig. 2).

$$
\begin{aligned}
\phi^{\prime} & =\frac{\left|\Omega_{m}^{\prime}\right|}{\left|\Omega_{s}^{\prime}\right|} \text { is the microporosity } \\
\langle\cdot\rangle_{\Omega^{\prime}} & =\frac{1}{\left|\Omega_{s}\right|} \int_{\Omega_{m}^{\prime}} \cdot \mathrm{d} \Omega \quad \text { is the average over the micropore periodic cell } \\
\langle\cdot\rangle_{\Omega} & =\frac{1}{|\Omega|} \int_{\Omega_{p}} \cdot \mathrm{d} \Omega \quad \text { is the average over the pore periodic cell }
\end{aligned}
$$

3.1.2. Governing equations. For the sake of simplicity, thermal effects are neglected in this part. The difference of both thermal regimes in the pores and micropores is accounted for by assuming the porous system to be in adiabatic conditions, and the miroporous system to be in isothermal conditions. Thus, the air state equation in the micropores is written as follows:

$$
P_{m}=\frac{P^{e}}{\rho^{e}} \rho_{m}
$$

Since the boundary of the grains, $\Gamma$, is not impervious, the adherence condition (4) is no longer valid. The actual boundary conditions must now express the continuity of pressure and flux (Auriault and Boutin, 1993-1994).

The governing equations are the following, where $k=m$ in the micropores and $k=p$ in the pores:

Momentum balance (Navier-Stokes equation)

$$
\mu \Delta \mathbf{v}_{k}+(\lambda+\mu) \boldsymbol{\nabla}\left(\boldsymbol{\nabla} \cdot \mathbf{v}_{k}\right)-\nabla P_{k}=\rho^{e} \frac{\partial \mathbf{v}_{k}}{\partial t}
$$

Mass balance (continuity equation)

$$
\frac{\mathrm{d} \rho_{k}}{\mathrm{~d} t}+\rho^{e} \boldsymbol{\nabla} \cdot \mathbf{v}_{k}=0
$$




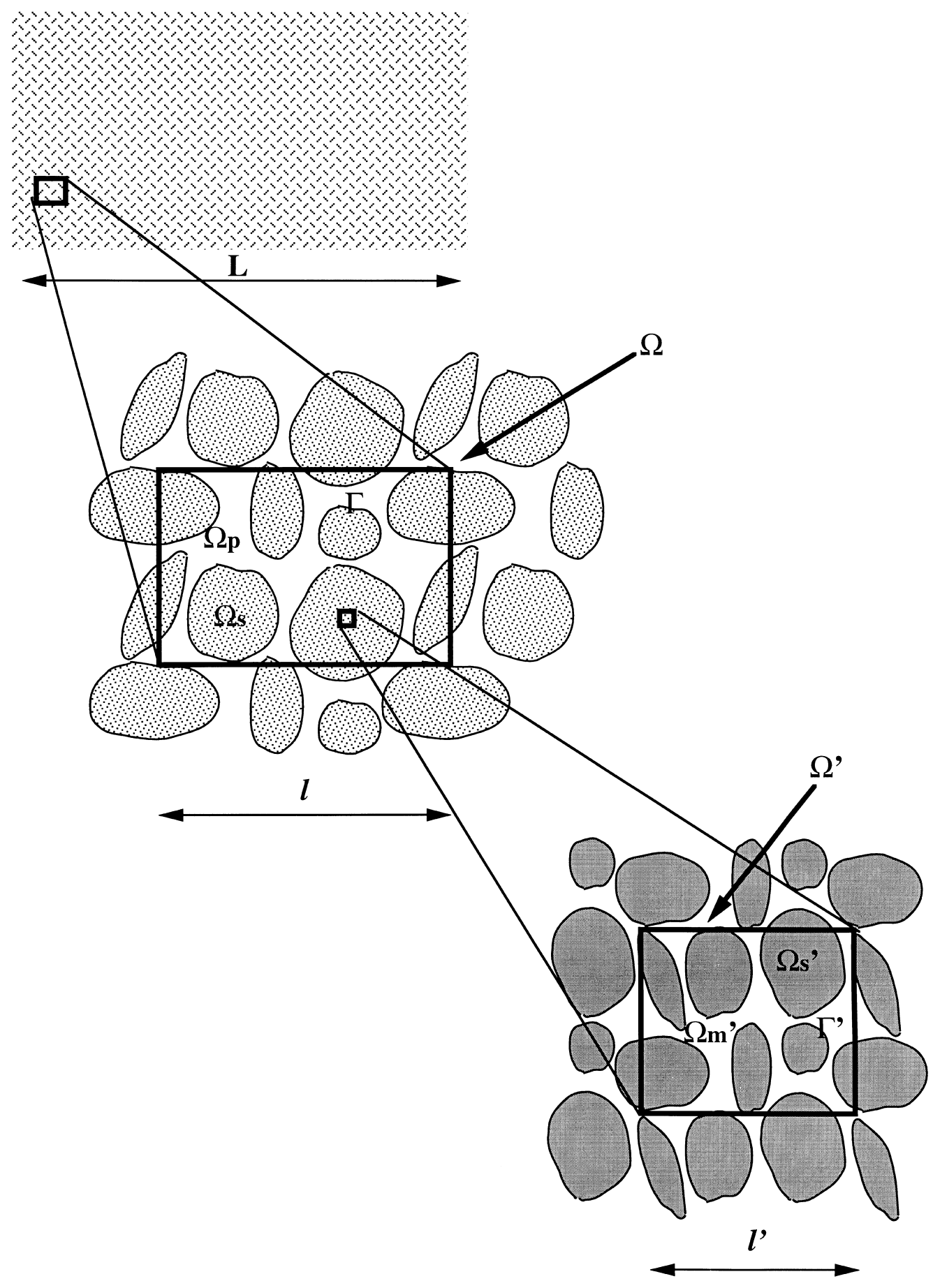

Fig. 2. Dual porosity medium (the grains are microporous) : description of both periodic cells at the pore scale and the micropore scale.

Air state equations

$$
\begin{gathered}
P_{p}=\frac{P^{e}}{\rho^{e}} \gamma \rho_{p} \\
P_{m}=\frac{P^{e}}{\rho^{e}} \rho_{m}
\end{gathered}
$$


Boundary conditions on $\Gamma$

$$
\begin{aligned}
\mathbf{v}_{p} / \Gamma & =\left\langle\mathbf{v}_{m}\right\rangle_{\Omega^{\prime}}=\frac{1}{\left|\Omega_{s}\right|} \int_{\Omega_{\Omega_{m}^{\prime}}} \mathbf{v}_{m} \mathrm{~d} \Omega \\
P_{p} & =P_{m}
\end{aligned}
$$

Adherence condition on $\Gamma^{\prime}$

$$
\mathbf{v}_{m} / \Gamma^{\prime}=\mathbf{0}
$$

3.1.3. Dimensionless numbers in the pores. Considering (36), the permeability of the microporous grains is smaller than the pore permeability. Thus, air velocity through the grains is small in comparison with air velocity between the grains. Therefore, the physics of a single porosity medium remains valid in the pores of a dual porosity medium. Hence, estimations made in Section 2.1.4 are still valid at the pore scale :

$$
Q_{p L}=O\left(\varepsilon^{-2}\right) \quad R t_{p L}=O\left(\varepsilon^{-2}\right) \quad S_{p L}=O(1)
$$

3.1.4. Dimensionless numbers in the micropores. We have now to estimate the dimensionless numbers for the microporous system, $Q_{m L}, R t_{m L}$ and $S_{m L}$. For this purpose, let us first describe the physics at this scale.

At its boundary, a microporous grain is submitted to a uniform harmonic pressure. Therefore, the continuity of stress does impose that:

$$
P_{m}=O\left(P_{p}\right)
$$

One the one hand, as a result of air compressibility, this pressure leads to a volume variation in all the micropores of a given grain. Thus:

$$
\omega P_{p}=O\left(\frac{P^{e} V_{m}}{l}\right)
$$

Using estimations in the pores gives the relative order of magnitude of velocities:

$$
V_{m}=O\left(\frac{\omega l P_{m}}{P^{e}}\right)=O\left(\frac{\omega l \rho_{p}}{\rho^{e}}\right)=O\left(V_{p} \frac{l}{L}\right)
$$

So, we have:

$$
\frac{\left|\mathbf{v}_{m}\right|}{\left|\mathbf{v}_{p}\right|}=O(\varepsilon)
$$

which expresses the fact that the air velocity is much greater in the pores than in the micropores. On the other hand, the flux in the micropores is dominated by viscous effects which induce a pressure gradient. The balance of these two terms gives:

$$
\frac{\mu V_{m}}{l^{2}}=O\left(\left|\nabla P_{m}\right|\right)
$$

so that 


$$
\left|\nabla P_{m}\right|=O\left(\frac{P}{l}\right)
$$

This means that the pressure is non-uniformly diffused in the microporous grains.

Using the previous physical analysis, we can now estimate the dimensionless numbers. Considering $L$ and $V_{p}$ as characteristic length and velocity, respectively, we deduce that:

$$
Q_{m L}=O\left[\frac{\frac{P}{L}}{\frac{\mu V_{p}}{L^{2}}}\right]=O\left(\varepsilon^{-2}\right)
$$

Since the transient Reynolds number has the same definition as at the pore scale, we have:

$$
R t_{m L}=O\left(\varepsilon^{-2}\right)
$$

Finally, the estimation of the Strouhal number in the micropores, is given by:

$$
\left|\rho^{e} \boldsymbol{\nabla} \cdot \mathbf{v}_{m}\right|=O\left(\frac{\rho^{e} V_{m}}{l}\right)=O(\omega \rho)
$$

Hence, we deduce :

$$
S_{m L}=O\left(\frac{\omega \rho}{\frac{\rho^{e} V_{p}}{L}}\right)=O(1)
$$

3.1.5. Dimensionless equations. Therefore, the dimensionless governing equations for harmonic pulsations are:

$$
\begin{gathered}
\varepsilon^{2} \mu \Delta \mathbf{v}_{k}+\varepsilon^{2}(\lambda+\mu) \nabla\left(\nabla \cdot \mathbf{v}_{k}\right)-\nabla P_{k}=i \omega \rho^{e} \mathbf{v}_{k} \\
i \omega \rho_{k}+\rho^{e} \nabla \cdot \mathbf{v}_{k}=0 \\
P_{p}=\frac{P^{e}}{\rho^{e}} \gamma \rho_{p} \\
P_{m}=\frac{P^{e}}{\rho^{e}} \rho_{m} \\
\mathbf{v}_{p} / \Gamma=\left\langle\mathbf{v}_{m}\right\rangle_{\Omega^{\prime}}=\frac{1}{\left|\Omega_{s}\right|} \int_{\Omega_{m}^{\prime}} \mathbf{v}_{m} \mathrm{~d} \Omega \\
P_{p}=P_{m} \quad \text { on } \Gamma \\
\mathbf{v}_{m} / \Gamma^{\prime}=\mathbf{0}
\end{gathered}
$$

In comparison with the single porosity case (see paragraph 2.1.3), we have to introduce a third dimensionless space variable for the micropores. 


$$
\mathbf{z}=\frac{\mathbf{X}}{l^{\prime}}
$$

The dimensionless gradient operator $\mathbf{\nabla}$ may now be written as follows:

$$
\boldsymbol{\nabla}:=\varepsilon^{-2} \boldsymbol{\nabla}_{z}+\varepsilon^{-1} \nabla_{y}+\nabla_{x}
$$

3.1.6. Homogenization. Each variable is looked for in the form of asymptotic expansions in power of $\varepsilon$ :

$$
\begin{aligned}
P_{m}(\mathbf{x}, \mathbf{y}, \mathbf{z}) & =\varepsilon^{0} P_{m}^{0}(\mathbf{x}, \mathbf{y}, \mathbf{z})+\varepsilon^{1} P_{m}^{1}(\mathbf{x}, \mathbf{y}, \mathbf{z})+\varepsilon^{2} P_{m}^{2}(\mathbf{x}, \mathbf{y}, \mathbf{z})+\cdots \\
\mathbf{v}_{m}(\mathbf{x}, \mathbf{y}, \mathbf{z}) & =\varepsilon^{1} \mathbf{v}_{m}^{1}(\mathbf{x}, \mathbf{y}, \mathbf{z})+\varepsilon^{2} \mathbf{v}_{m}^{2}(\mathbf{x}, \mathbf{y}, \mathbf{z})+\cdots \\
\rho_{m}(\mathbf{x}, \mathbf{y}, \mathbf{z}) & =\varepsilon^{0} \rho_{m}^{0}(\mathbf{x}, \mathbf{y}, \mathbf{z})+\varepsilon^{1} \rho_{m}^{1}(\mathbf{x}, \mathbf{y}, \mathbf{z})+\varepsilon^{2} \rho_{m}^{2}(\mathbf{x}, \mathbf{y}, \mathbf{z})+\cdots \\
P_{p}(\mathbf{x}, \mathbf{y}) & =\varepsilon^{0} P_{p}^{0}(\mathbf{x}, \mathbf{y})+\varepsilon^{1} P_{p}^{1}(\mathbf{x}, \mathbf{y})+\varepsilon^{2} P_{p}^{2}(\mathbf{x}, \mathbf{y})+\cdots \\
\mathbf{v}_{p}(\mathbf{x}, \mathbf{y}) & =\varepsilon^{0} \mathbf{v}_{p}^{0}(\mathbf{x}, \mathbf{y})+\varepsilon^{1} \mathbf{v}_{p}^{1}(\mathbf{x}, \mathbf{y})+\varepsilon^{2} \mathbf{v}_{p}^{2}(\mathbf{x}, \mathbf{y})+\cdots \\
\rho_{p}(\mathbf{x}, \mathbf{y}) & =\varepsilon^{0} \rho_{p}^{0}(\mathbf{x}, \mathbf{y})+\varepsilon^{1} \rho_{p}^{1}(\mathbf{x}, \mathbf{y})+\varepsilon^{2} \rho_{p}^{2}(\mathbf{x}, \mathbf{y})+\cdots
\end{aligned}
$$

All these quantities are $\Omega$-periodic, with respect to the variable $\mathbf{y}$. The quantities related to the micropores are also $\Omega^{\prime}$-periodic, with respect to the variable $\mathbf{z}$. Introducing these expansions in the set of eqns (40)-(46), gives the following governing equations at the first significant orders.

In the micropores

Equation (4) and $O\left(\varepsilon^{-2}\right)$ and $O\left(\varepsilon^{-1}\right)$

$$
\begin{gathered}
\boldsymbol{\nabla}_{z} P_{m}^{0}=\mathbf{0} \\
\mu \Delta_{z} \mathbf{v}_{m}^{1}+(\lambda+\mu) \nabla_{z}\left(\nabla_{z} \cdot \mathbf{v}_{m}^{1}\right)-\nabla_{z} P_{m}^{1}-\nabla_{y} P_{m}^{0}=\mathbf{0}
\end{gathered}
$$

Equation (41) at $O\left(\varepsilon^{-1}\right)$ and $O\left(\varepsilon^{0}\right)$

$$
\begin{gathered}
\boldsymbol{\nabla}_{z} \cdot \mathbf{v}_{m}^{1}=0 \\
i \omega \rho^{0}+\rho^{e} \boldsymbol{\nabla}_{z} \cdot \mathbf{v}_{m}^{2}+\rho^{e} \nabla_{y} \cdot \mathbf{v}_{m}^{1}=0
\end{gathered}
$$

Equation (43) at $O\left(\varepsilon^{0}\right)$ and $O\left(\varepsilon^{1}\right)$

$$
\begin{gathered}
P_{m}^{0}=\frac{P^{e}}{\rho^{e}} \rho_{m}^{0} \\
P_{m}^{1}=\frac{P^{e}}{\rho^{e}} \rho_{m}^{1}
\end{gathered}
$$


On the boundary $\Gamma^{\prime}$

Equation (46) at $O\left(\varepsilon^{1}\right)$ and $O\left(\varepsilon^{2}\right)$

$$
\begin{aligned}
& \mathbf{v}_{m}^{1} / \Gamma^{\prime}=\mathbf{0} \\
& \mathbf{v}_{m}^{2} / \Gamma^{\prime}=\mathbf{0}
\end{aligned}
$$

In the pores

Apart from the boundary conditions, we obtain the same set of equations as in the case of single porosity. The only difference is that variables are now indexed by $p$. Navier-Stokes equation (40) at $O\left(\varepsilon^{-1}\right)$ and $O\left(\varepsilon^{0}\right)$ leads to (9) and (10), respectively. Mass balance eqn (41) at $O\left(\varepsilon^{-1}\right)$ and $O\left(\varepsilon^{0}\right)$ gives (11) and (12). Equation (42) at $O\left(\varepsilon^{0}\right)$ corresponds to (13).

On the boundary $\Gamma$

Equation (45) at $O\left(\varepsilon^{0}\right)$ and $O\left(\varepsilon^{1}\right)$

$$
\begin{aligned}
& P_{p}^{0}=P_{m}^{0} \\
& P_{p}^{1}=P_{m}^{1}
\end{aligned}
$$

Equation (44) at $O\left(\varepsilon^{0}\right)$ and $O\left(\varepsilon^{1}\right)$

$$
\begin{gathered}
\mathbf{v}_{p}^{0} /_{\Gamma}=\left\langle\mathbf{v}_{m}^{0}\right\rangle_{\Omega^{\prime}}=\mathbf{0} \\
\mathbf{v}_{p}^{1} /_{\Gamma}=\left\langle\mathbf{v}_{m}^{1}\right\rangle_{\Omega^{\prime}}
\end{gathered}
$$

The approach to the solution comprises three stages. The flow in micropores and pores are successively analysed and finally the coupling flux between both scales is derived.

In the micropores

Equations (47) and (51) give:

$$
\begin{aligned}
P_{m}^{0} & =P_{m}^{0}(\mathbf{y}, \mathbf{x}) \\
\rho_{m}^{0} & =\rho_{m}^{0}(\mathbf{y}, \mathbf{x})
\end{aligned}
$$

Equations (48), (49) and (53) lead to a classical steady-state Darcy's flow in the micropores:

$$
\mathbf{v}_{m}^{1}=-\frac{\tilde{k}_{m}}{\mu} \nabla_{y} P_{m}^{0}
$$

This set of equations also determines $P_{m}^{1}$ and then $\rho_{m}^{1}$ is defined by eqn (52).

Integration of (50) over the micropore period yields the mass balance in this domain:

$$
i \omega\left\langle\rho_{m}^{0}\right\rangle_{\Omega^{\prime}}+\rho^{e} \nabla_{y} \cdot\left\langle\mathbf{v}_{m}^{1}\right\rangle_{\Omega^{\prime}}=0
$$

Now, using eqn (51) and averaging eqn (59) gives the equation governing the pressure variation in $\Omega_{s}$ :

$$
\frac{\phi^{\prime} i \omega}{P^{e}} P_{m}^{0}-\nabla_{y} \cdot\left(\frac{\widetilde{K}_{m}}{\mu} \nabla_{y} P_{m}^{0}\right)=0
$$

where $\tilde{K}_{m}=\left\langle\tilde{k}_{m}\right\rangle_{\Omega^{\prime}}$ is the steady flow intrinsic permeability of the microporous system. 
In the pores

The resolution process is exactly the same as in Section 2.1.5. We successively get:

$$
\begin{gathered}
P_{p}^{0}=P_{p}^{0}(\mathbf{x}) \\
\rho_{p}^{0}=\rho_{p}^{0}(\mathbf{x}) \\
\mathbf{v}_{p}^{0}=-\frac{\tilde{k}_{p}}{\mu} \nabla_{x} P_{p}^{0}
\end{gathered}
$$

However, the macroscopic mass balance obtained by integrating eqn (12) over $\Omega_{p}$, gives now:

$$
i \omega\left\langle\rho_{p}^{0}\right\rangle_{\Omega}+\rho^{e}\left\langle\nabla_{y} \cdot \mathbf{v}_{p}^{1}\right\rangle_{\Omega}+\rho^{e} \boldsymbol{\nabla}_{x} \cdot\left\langle\mathbf{v}_{p}^{0}\right\rangle_{\Omega}=0
$$

which can also be written:

$$
\frac{\phi i \omega}{P^{e} \gamma} P_{p}^{0}+\left\langle\nabla_{y} \cdot \mathbf{v}_{p}^{1}\right\rangle_{\Omega}-\nabla_{x} \cdot\left(\frac{\tilde{K}_{p}}{\mu} \nabla_{x} P_{p}^{0}\right)=0
$$

In order to determine the additional term due to $\mathbf{v}_{p}^{1}$, we must go back to the micropore scale. The actual pressure distribution in $\Omega_{s}$ is defined by eqns (61), (62) and (55), which give the following boundary-value problem:

$$
\begin{gathered}
\frac{\phi^{\prime} i \omega}{P^{e}} P_{m}^{0}-\nabla_{y} \cdot\left(\frac{\tilde{K}_{m}}{\mu} \nabla_{y} P_{m}^{0}\right)=0 \quad \text { in } \Omega_{s} \\
P_{m}^{0}=P_{p}^{0}(\mathbf{x}) \quad \text { on } \Gamma
\end{gathered}
$$

Using the pressure difference, this problem can be rewritten in the following equivalent form:

$$
\begin{gathered}
P^{\prime}=P_{m}^{0}-P_{p}^{0} \\
\frac{\phi^{\prime} i \omega}{P^{e}}\left(P^{\prime}+P_{p}^{0}\right)-\nabla_{y} \cdot\left(\frac{\tilde{K}_{m}}{\mu} \nabla_{y} P^{\prime}\right)=0 \text { in } \Omega_{s} \\
P^{\prime}=0 \text { on } \Gamma
\end{gathered}
$$

It turns out that the pressure difference is governed by a diffusion equation with a forcing term. Note that this boundary value problem looks like the heat transfer problem encountered in eqns (27)-(28) for the temperature field. We deduce that the pressure fields in the micropores and in the pores are related by:

$$
P^{\prime}=-\pi P_{p}^{0}
$$

where the function $\pi(\mathbf{y})$ is a complex, depends on the local variable $\mathbf{y}$ and on the dimensionless pulsation $\omega / \omega_{d}$, where $\omega_{d}$ is a characteristic diffusion pulsation, which is of the order of $K_{m} P^{e} / l^{2} \mu \phi^{\prime}$. Then, equation (61) can be written as follows : 


$$
\frac{\phi^{\prime} i \omega}{P^{e}}(1-\pi) P_{p}^{0}-\nabla_{y} \cdot \mathbf{v}_{m}^{1}=0
$$

Finally, the expression of $\left\langle\nabla_{y} \cdot \mathbf{v}_{p}^{1}\right\rangle_{\Omega}$ is derived by considering the boundary condition (58) and integrating (66) over $\Omega_{s}$ :

$$
\left\langle\nabla_{y} \cdot \mathbf{v}_{p}^{1}\right\rangle_{\Omega}=\frac{1}{|\Omega|} \int_{\Gamma}\left\langle\mathbf{v}_{m}^{1}\right\rangle_{\Omega^{\prime}} \cdot \mathbf{n} \mathrm{d} S=\phi^{\prime}(1-\phi) \frac{i \omega}{P^{e}} P_{p}^{0}(1-\Pi)
$$

where

$$
\Pi=\frac{1}{\left|\Omega_{s}\right|} \int_{\Omega_{s}} \pi(\mathbf{y}) \mathrm{d} \Omega
$$

Thus, the macroscopic description is given by:

$$
\left[\phi+\gamma \phi^{\prime}(1-\phi)(1-\Pi)\right] \frac{i \omega}{\gamma P^{e}} P_{p}^{0}-\nabla_{x} \cdot\left(\frac{\tilde{K}_{p}}{\mu} \nabla_{x} P_{p}^{0}\right)=0
$$

3.1.7. Comments. The difference between the dual porosity model (67) and the single porosity model (20) consists of a modification of the porosity to a new complex valued term. This latter corresponds to the influence of air saturating the whole micropores of the grains. It highlights a coupling effect between pore and micropore air fluxes, which is due to the diffusion of the pore pressure in the micropores. This phenomenon is expressed by the complex valued function $\Pi$, which depends on the dimensionless pulsation $\omega_{d}^{*}=\omega / \omega_{d}$, where the characteristic diffusion pulsation $\omega_{d}$ is of the order of $K_{m} P^{e} / l^{2} \mu \phi^{\prime}$. As observed above, the pressure diffusion problem is exactly of the same kind as the heat transfer problem. Therefore the properties established for $G$ are also valid for $\Pi$.

At "low" frequencies, the transient diffusion effects are negligible. Therefore, the micropore pressure is uniformly equal to the pore pressure, so that $\Pi(0)=0$ and the total gas volume contributes to the compressibility. Note that the dynamic Darcy's law affects the pore volume only. As a result, this description does not reduce to a single porosity.

At "high" frequencies the diffusion affects air only in the vicinity of the pore walls. The pressure does not vary in the micropores, which gives $\Pi(\infty)=1$. We recognise the single porosity behaviour.

At "medium" frequencies, i.e. for pulsations of the order of $\omega_{d}$, there is a phase shift between pressure and velocity in the micropores, leading to a complex value for $\Pi$. As for $\omega_{t}$, an improved expression of $\omega_{d}$ can be obtained by introducing the length $\Lambda_{d}$ defined as the ratio of the grain volume to the surface:

$$
\omega_{d}=\frac{K_{m} P^{e}}{\Lambda_{d}^{2} \mu \phi^{\prime}} \quad \Lambda_{d}=\frac{\left|\Omega_{s}\right|}{|\Gamma|}
$$

Obviously, $\Lambda_{t}$ and $\Lambda_{d}$ are related by:

$$
\Lambda_{d}=(1-\phi) \Lambda_{t} / \phi
$$

The physical meaning of this characteristic pulsation is that, when $\omega=\omega_{d}$, the thickness of the diffusion layer $\left(\sqrt{K_{m} P^{e} / \omega \mu \phi^{\prime}}\right)$ equals the grain size. Let us notice that we have : 


$$
\sqrt{\frac{\omega_{d}}{\omega_{c}}}=\frac{\sqrt{K K_{m}}}{\Lambda_{d}} \sqrt{\frac{P^{e} \rho^{e}}{\mu^{2} \phi \phi^{\prime}}}=O\left(l^{\prime} \frac{\sqrt{P^{e} \rho^{e}}}{\mu}\right)
$$

In air, $\mu / \sqrt{P^{e} \rho^{e}} \approx 10^{-7} \mathrm{~m}$, and consequently, for usual values of the micropore sizes :

$$
\omega_{d}>\omega_{c}
$$

Therefore, at the macroscopic scale, a new dissipation effect appears at higher frequencies. For the same reasons as for thermal dissipation, the influence of diffusion is limited in the frequency range and its effects are maximum for pulsations close to $\omega_{d}$.

Finally, like $G, \Pi$ may be expressed analytically for spherical or cylindrical grains. For other micropore geometries, the expression below gives the correct asymptotic behaviour at low and large frequencies:

$$
\Pi\left(\omega_{d}^{*}\right)=\left[1+\sqrt{F_{d}^{2}+\omega_{d}^{*}} / i \omega_{d}^{*}\right]^{-1}
$$

where $F_{d}$ is the shape ratio of the grain structure ( $F^{\prime \prime}=2$ for cylindrical grains and $F_{d}=5 / 3$ for spherical grains).

In isotropic cases, the expression of the complex valued acoustic velocity is :

$$
C^{2}=C_{a}^{2}\left(\frac{i K^{*} \omega^{*}}{\alpha_{\infty}}\right)\left[1+\frac{(1-\phi) \phi^{\prime}}{\phi} \gamma(1-\Pi)\right]^{-1}
$$

The comparison with eqn (23) - which is valid for single porosity media under adiabatic conditions - shows that this complex valued celerity presents the same behaviour at high frequencies $\left(\omega \gg \omega_{d}\right)$, but is augmented by a factor $\sqrt{1+\left[(1-\phi) \phi^{\prime} / \phi\right] \gamma}$ at low frequencies $\left(\omega \ll \omega_{d}\right)$. For the same reasons as for the thermal dissipation, the influence of the microporosity on the attenuation is maximum for pulsations close to $\omega_{d}$.

\subsection{Adding thermal effects}

The preceding description may be improved by considering thermal exchanges. As in the case of single porosity, thermal effects do not act on the description of the flow, but only modify mass balances. Therefore, the results obtained in the treatment of NavierStokes equations in Section 3.1 are still valid here.

3.2.1. Macroscopic behaviour. In the pores and in the micropores, the governing equations for heat transfer are the same as (24)-(25), but variables are now indexed by $p$ or $m$.

In comparison with the single porosity case, the description of thermal exchanges in the pores is unchanged, i.e., the thermal skin is of the order of the pore size. Therefore, as in Section 2.2., we have:

$$
N_{p L}=O\left(\varepsilon^{-2}\right)
$$

Due to the separation of scale, the micropore size is smaller than the thermal skin:

$$
l^{\prime}=O\left(\varepsilon \delta_{v}\right)
$$


The continuity of heat flux at the grain boundary implies that:

$$
\kappa \frac{T_{p}}{l}=\kappa \frac{T_{m}}{l^{\prime}}
$$

which shows that:

$$
T_{m}=O\left(\varepsilon T_{p}\right)
$$

Now, in the solid the level of temperature is also given by the heat flux continuity:

$$
\kappa_{s} \frac{T_{s}}{l^{\prime}}=\kappa \frac{T_{m}}{l^{\prime}}
$$

Because of the contrast in conductivities, it can be assumed, for simplicity, that the solid remains in isothermal conditions (note that this hypothesis could be modified without difficulties). This analysis leads to quasi-static exchanges at the microscopic scale. However, in order to treat the largest frequency domain as possible, we keep in the transient terms at the micropore scale. So we have:

$$
N_{m L}=O\left(N_{p L}\right) \times \varepsilon^{-1}=O\left(\varepsilon^{-3}\right)
$$

These estimations yield the following scaled equations, where $L$ and $T_{p}$ are used as references quantities :

$$
\begin{gathered}
\varepsilon^{3} \nabla \cdot\left(\kappa \nabla T_{m}\right)=i \omega\left(\rho^{e} c_{p} T_{m}-\varepsilon P_{m}\right) \\
\varepsilon^{2} \nabla \cdot\left(\kappa \nabla T_{p}\right)=i \omega\left(\rho^{e} c_{p} T_{p}-P_{p}\right) \\
P_{m}=P^{e}\left(\frac{\rho_{m}}{\rho^{e}}+\varepsilon^{-1} \frac{T_{m}}{T^{e}}\right) \\
P_{p}=P^{e}\left(\frac{\rho_{p}}{\rho^{e}}+\frac{T_{p}}{T^{e}}\right) \\
T_{m} / \Gamma^{\prime}=0 \\
T_{p}=T_{m} \quad \text { on } \Gamma
\end{gathered}
$$

The temperature fields are looked for in the form of asymptotic expansions:

$$
\begin{aligned}
T_{p}(\mathbf{x}, \mathbf{y}) & =\varepsilon^{0} T_{p}^{0}(\mathbf{x}, \mathbf{y})+\varepsilon^{1} T_{p}^{1}(\mathbf{x}, \mathbf{y})+\varepsilon^{2} T_{p}^{2}(\mathbf{x}, \mathbf{y})+\cdots \\
T_{m}(\mathbf{x}, \mathbf{y}, \mathbf{z}) & =\varepsilon^{1} T_{m}^{1}(\mathbf{x}, \mathbf{y}, \mathbf{z})+\varepsilon^{2} T_{m}^{2}(\mathbf{x}, \mathbf{y}, \mathbf{z})+\cdots
\end{aligned}
$$

Firstly, the influence of thermal effects is derived by establishing the mass balance at both scales, and then by determining the inter-scale coupling term. 
The following problems must be solved:

In the micropores:

$$
\begin{aligned}
\nabla_{z} \cdot\left(\frac{\kappa}{i \omega \rho^{e} c_{p}} \frac{T_{m}^{1}}{T^{e}}\right)-\frac{T_{m}^{1}}{T^{e}} & =-\left(1-\frac{1}{\gamma}\right) \frac{P_{m}^{0}}{P^{e}} \\
T_{m}^{1} / \Gamma^{\prime} & =0
\end{aligned}
$$

In the pores:

$$
\begin{aligned}
& \nabla_{y} \cdot\left(\frac{\kappa}{i \omega \rho^{e} c_{p}} \frac{T_{p}^{0}}{T^{e}}\right)-\frac{T_{p}^{0}}{T^{e}}=-\left(1-\frac{1}{\gamma}\right) \frac{P_{p}^{0}}{P^{e}} \\
& T_{p}^{0} / \Gamma=0
\end{aligned}
$$

Both problems are similar to that defined by eqns (24) and (25) for the single porosity problem.

The solutions are:

$$
\begin{aligned}
& T_{m}^{1}=\frac{T^{e}}{P^{e}}(1-\gamma) g_{m} P_{m}^{0} \\
& T_{p}^{0}=\frac{T^{e}}{P^{e}}(1-\gamma) g_{p} P_{p}^{0}
\end{aligned}
$$

Now, considering eqns (71) and (72) at the first order yields:

$$
\begin{aligned}
& \frac{\rho_{m}^{0}}{\rho^{e}}=\frac{P_{m}^{0}}{P^{e}}-\frac{T_{m}^{1}}{T^{e}}=\left[1-\left(1-\frac{1}{\gamma}\right) g_{m}\right] \frac{P_{m}^{0}}{P^{e}} \\
& \frac{\rho_{p}^{0}}{\rho^{e}}=\frac{P_{p}^{0}}{P^{e}}-\frac{T_{p}^{0}}{T^{e}}=\left[1-\left(1-\frac{1}{\gamma}\right) g_{p}\right] \frac{P_{p}^{0}}{P^{e}}
\end{aligned}
$$

which give for the average density variations :

$$
\begin{gathered}
\left\langle\rho_{m}^{0}\right\rangle_{\Omega^{\prime}}=\rho^{e}\left[1-\left(1-\frac{1}{\gamma}\right) G_{m}\right] \frac{P_{m}^{0}}{P^{e}} \\
\left\langle\rho_{p}^{0}\right\rangle_{\Omega}=\rho^{e}\left[1-\left(1-\frac{1}{\gamma}\right) G_{p}\right] \frac{P_{p}^{0}}{P^{e}} \\
G_{m}=\frac{1}{\left|\Omega_{m}^{\prime}\right|} \int_{\Omega_{m}^{\prime}} g_{m} \mathrm{~d} \Omega \quad G_{p}=\frac{1}{|\Omega|} \int_{\Omega_{p}} g_{p} \mathrm{~d} \Omega
\end{gathered}
$$

These complex valued functions $G_{m}$ and $G_{p}$ play exactly the same role as the function $G$ in the single porosity case. Each of them is associated with a characteristic pulsation given by:

$$
\omega_{t p}=\frac{\kappa}{\Lambda_{t p}^{2} \rho^{e} c_{p}} \quad \Lambda_{t p}=\frac{\left|\Omega_{p}\right|}{|\Gamma|} \quad \omega_{t m}=\frac{\kappa}{\Lambda_{t m}^{2} \rho^{e} c_{p}} \quad \Lambda_{t m}=\frac{\left|\Omega_{m}\right|}{\left|\Gamma^{\prime}\right|}
$$

The descriptions of the flow in the pores (59) and in the micropores (64) remain valid. But, 
considering equations (75)-(76), the mass balances (60)-(65) for the micropores and pores, respectively, become:

$$
\begin{gathered}
\phi^{\prime} i \omega\left[1-\left(1-\frac{1}{\gamma}\right) G_{m}\right] \frac{P_{m}^{0}}{P^{e}}+\nabla_{y} \cdot\left\langle\mathbf{v}_{m}^{1}\right\rangle_{\Omega^{\prime}}=0 \\
i \omega\left\langle\rho_{p}^{0}\right\rangle_{\Omega}+\rho^{e}\left\langle\nabla_{y} \cdot \mathbf{v}_{p}^{1}\right\rangle_{\Omega}+\rho^{e}\left\langle\mathbf{v}_{p}^{0}\right\rangle_{\Omega}=0
\end{gathered}
$$

Let us now determine the inter-scale coupling term. Equation (77) with the boundary condition:

$$
P_{m}^{0}=P_{p}^{0} \quad \text { on } \Gamma
$$

constitutes a boundary value problem in $\Omega_{s}$. This problem looks like the pressure diffusion problem encountered in the preceding section [eqns (55) and (61)]. However, due to the thermal non-equilibrium in the micropores, the diffusion coefficient is now complex and frequency dependent. The solution is :

$$
P_{m}^{0}=(1-\psi) P_{p}^{0}
$$

where $\psi$ is complex and depends on $\mathbf{y}$ and also on the pulsation $\omega$ and on both characteristic pulsations $\omega_{m}$ and $\omega_{d}$.

As for $\left\langle\nabla_{y} \cdot \mathbf{v}_{p}^{1}\right\rangle_{\Omega}$, it is derived using boundary condition (58), and integrating (77) over $\Omega_{s}$ while considering expression (59):

$$
\left\langle\nabla_{y} \cdot \mathbf{v}_{p}^{1}\right\rangle_{\Omega}=i \omega\left[1-\left(1-\frac{1}{\gamma}\right) G_{m}\right](1-\Psi) \frac{P_{p}^{0}}{P^{e}}
$$

where

$$
\Psi=\frac{1}{\left|\Omega_{s}\right|} \int_{\Omega_{s}} \psi \mathrm{d} \Omega
$$

Finally, the macroscopic behaviour is :

$$
\left\{\phi\left[1-\left(1-\frac{1}{\gamma}\right) G_{p}\right]+\phi^{\prime}(1-\phi)\left[1-\left(1-\frac{1}{\gamma}\right) G_{m}\right](1-\Psi)\right\} \frac{i \omega}{P^{e}} P_{p}^{0}-\nabla_{x} \cdot\left(\frac{\tilde{K}_{p}}{\mu} \nabla_{x} P_{p}^{0}\right)=0
$$

3.2.2. Comments. Adding thermal effects alters description (67) by modifying the interscale coupling term. Both thermal and pressure diffusion effects are now involved at the micropore scale, which is particularly highlighted through the function $\Psi$ in which both phenomena are mixed.

Thermal exchanges in the micropores introduce a new characteristic frequency $\omega_{t m}^{*}$ and a function $G_{m}$ whose physical meaning corresponds to those of $\omega_{t}^{*}$ and function $G$ for the pores, respectively. The expression given by equation (34) is valid when $G$ is replaced by $G_{m}$ and $\omega_{t}^{*}$ by $\omega_{t m}^{*}$.

Although $\Pi$ and $\Psi$ are different, they have the same limit values, $\Psi(0)=0$ and $\Psi(\infty)=1$, and the same characteristics pulsation, $\omega_{d}=K_{m} P^{e} / \Lambda_{d}^{2} \mu \phi^{\prime}$. However, for pulsations higher than $\omega_{t m}$, the behaviours of $\Pi$ and $\Psi$ are different because of the complex valued diffusion coefficient. An expression which gives the correct asymptotic behaviour at low and high frequencies is given below: 


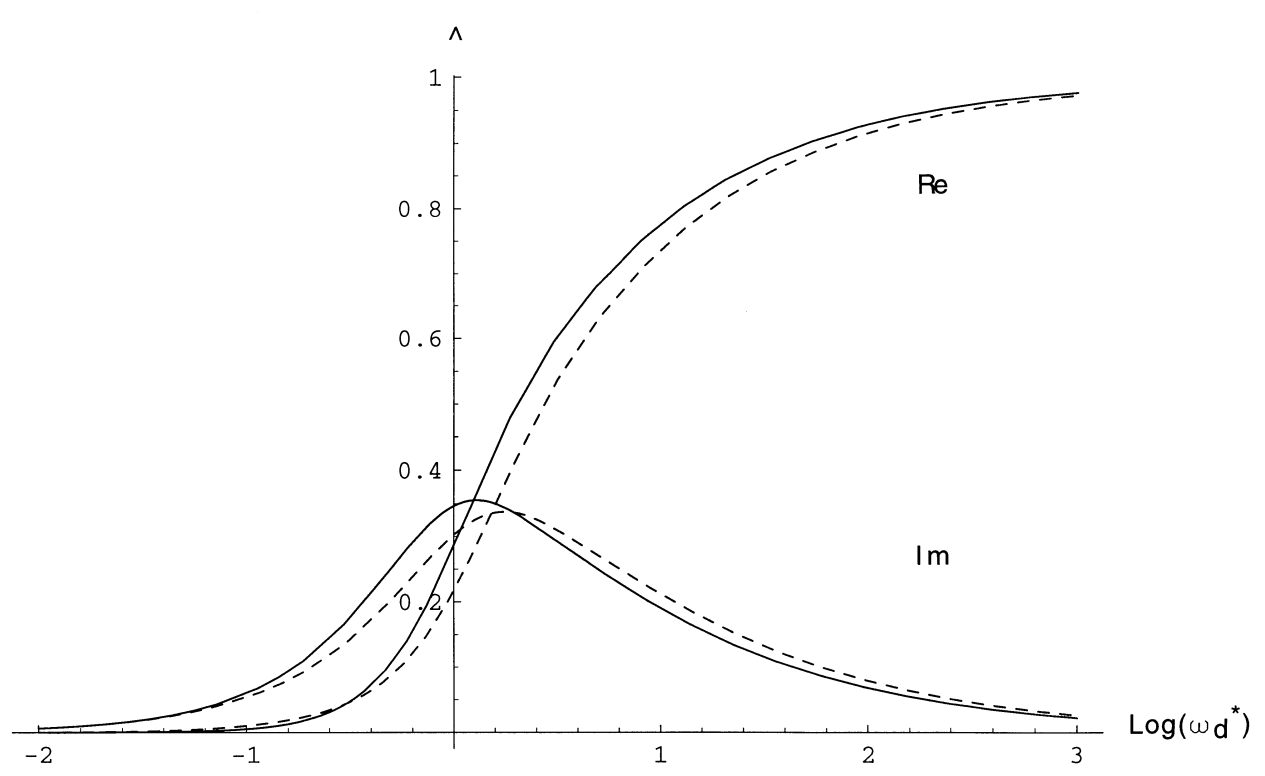

Fig. 3. Real and imaginary parts of the complex-valued functions $\Pi$ (diffusion) and $\Psi$ (thermodiffusion), with respect to the dimensionless diffusion pulsation $\omega_{d}^{*}$. Solid line : function $\Pi$. Dashed line: function $\Psi$.

$$
\Psi\left(\omega_{d}^{*}\right)=\left[1+\sqrt{F_{d}^{2}+i \Omega_{d}^{*}} / i \Omega_{d}^{*}\right]^{-1}
$$

where

$$
\Omega_{d}^{*}=\omega_{d}^{*} /\left[1-\left(1-\frac{1}{\gamma}\right) G_{m}\left(\omega_{t m}^{*}\right)\right]
$$

Figure 3 shows the functions $\Pi$ and $\Psi$ with respect to the pulsation $\omega_{d}$ for spherical grains. From eqn (80) we deduce that for isotropic cases, the acoustic velocity is expressed by:

$$
C^{2}=C_{a}^{2} \frac{i K^{*} \omega^{*}}{\alpha_{\infty}}\left[(\gamma-(\gamma-1)) G_{p}+\frac{(1-\phi) \phi^{\prime}}{\phi}(\gamma-(\gamma-1)) G_{m}(1-\psi)\right]^{-1}
$$

In comparison with the acoustic velocity for the single porosity case (35), these results show an increased dissipation at frequencies close to $\omega_{d}$ and $\omega_{t m}$.

\section{CONCLUSIONS}

In this study, various macroscopic descriptions of sound propagation through a rigid porous medium saturated by air are derived using homogenization theory. The results presented are valid as long as the wavelength is large in comparison with the pore size.

The first part of the paper deals with single porosity materials. The case of large pores, for which thermal exchanges are negligible [equations (20)-(23)] and then the case of small pores where thermal effects must be considered [eqns (33)-(35)] have been successively investigated. The derived descriptions are the same as those already obtained via phenomenological approaches (Allard, 1993; Attenborough, 1983). Air flow is governed by a dynamic Darcy's law where both viscous and inertial effects act, and the effective compressibility is influenced by thermal exchanges.

In the second part, we focus on dual porosity media, i.e. media in which the grains of the skeleton are microporous. Let us notice that the results could also be applied to granular media that consist of grains of very different sizes. 
Firstly, a simplified macroscopic description is obtained by neglecting heat transfer effects (67). We demonstrate that, in the micropores, the physics of the flow is very different to that in the pores. Due to the weak micropore permeability and to air compressibility, the pressure is inhomogeneously diffused in the micropores. This phenomenon is associated with a characteristic pulsation $\omega_{d}$, which is greater than the classical thermal and critical pulsations $\left(\omega_{t}, \omega_{c}\right)$, that are defined for single porosity media. It is shown that the effect of pressure diffusion implies increased acoustic attenuation for pulsations of the order of magnitude of $\omega_{d}$.

When thermal transfers are included (80), a second thermal characteristic pulsation associated with the micropore appears in the model, $\omega_{t m}$, which is greater than $\omega_{t}$. This increases acoustic attenuation for pulsations close to $\omega_{t m}$.

As a first example, let us determine the different characteristic pulsations for several dual porosity media.

We consider a medium similar to porous road surfacing having a porosity, $\phi=0.25$, a mean pore radius $R$ of about $5 \times 10^{-3} \mathrm{~m}$, an intrinsic permeability $K=10^{-9} \mathrm{~m}^{2}$, and a tortuosity of $\alpha_{\infty}=1.5$.

The limit frequency for which waves are diffracted on the pores is reached when the wavelength equals $2 \pi R$. In this specific case, one obtains a range of validity between 0 and $8 \mathrm{kHz}$ for the description.

The numerical values lead to the following characteristic frequencies for the pores:

$$
\begin{aligned}
& f_{c}=\frac{\omega_{c}}{2 \pi}=\frac{\mu \phi}{2 \pi \alpha_{\infty} K(0) \rho} \approx 430 \mathrm{~Hz} \\
& f_{t}=\frac{\omega_{t}}{2 \pi}=\frac{\kappa}{2 \pi \Lambda_{t}^{2} \rho^{e} c_{\mathrm{p}}} \approx 1.2 \mathrm{~Hz}
\end{aligned}
$$

Therefore, in the context of acoustics, the adiabatic approximation is valid for this material, and the dissipation is mainly due to the viscosity.

Consider now the case where the grains of this material have also an open microporosity, $\phi^{\prime}=0.25$, and the micropores are twenty times smaller than pores so that the mean micropore radius is about $2.5 \times 10^{-4} \mathrm{~m}$. The intrinsic permeability can be estimated as $K_{m}=2.5 \times 10^{-12} \mathrm{~m}^{2}$. These values lead to the following microporous characteristics frequencies :

$$
\begin{gathered}
f_{d}=\frac{\omega_{d}}{2 \pi}=\frac{K_{m} P^{e}}{2 \pi \Lambda_{d}^{2} \mu \phi^{\prime}} \approx 5.1 \mathrm{kHz} \\
f_{t m}=\frac{\omega_{t m}}{2 \pi}=\frac{\kappa}{2 \pi \Lambda_{t m}^{2} \rho^{e} c_{\mathrm{p}}} \approx 480 \mathrm{~Hz}
\end{gathered}
$$

These frequencies belong to the acoustic domain. Therefore, one can expect two peaks of attenuation (at these two frequencies).

Figure 4 shows the celerity with respect to the pulsation in such a medium. The influence of the microporosity appears through the presence of a second peak. For comparison, the celerities of two distinct single porosity media are presented: (i) the porosity is the pore porosity only; (ii) the porosity is the global porosity. Note that these two single porosity media and the dual porosity medium have the same static permeability and the same tortuosity coefficient. These curves show significant differences between the models. As a consequence, the three impedances are also different.

As a second example, consider the situation where we would like to increase the absorption for frequencies of about $1 \mathrm{kHz}$. With the same open microporosity $\phi^{\prime}=0.25$, a micropore radius of about $10^{-4} \mathrm{~m}$ can be chosen. The intrinsic permeability can be estimated as $K=4 \times 10^{-13} \mathrm{~m}^{2}$. The values of microporous characteristic frequencies become : 


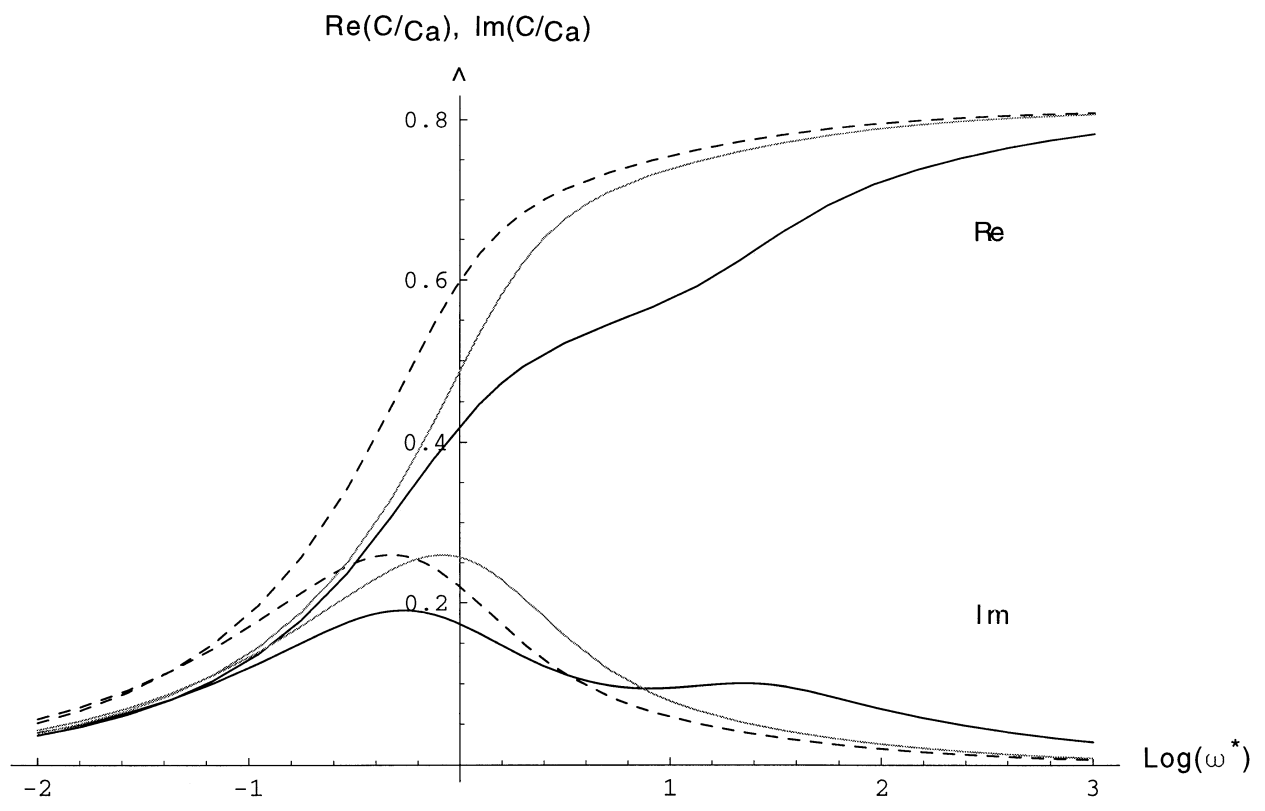

Fig. 4. Comparison between single porosity and dual porosity media. Real and imaginary parts of complex-valued celerities, with respect to the dimensionless pulsation $\omega^{*}$. Thick line : celerity of a dual porosity medium (model given in 3.2, with numerical values considered in example 1 in the conclusion). Thin line: celerity of a single porosity medium (pore porosity only). Dashed line: celerity of a single porosity medium (pore plus micropore porosity).

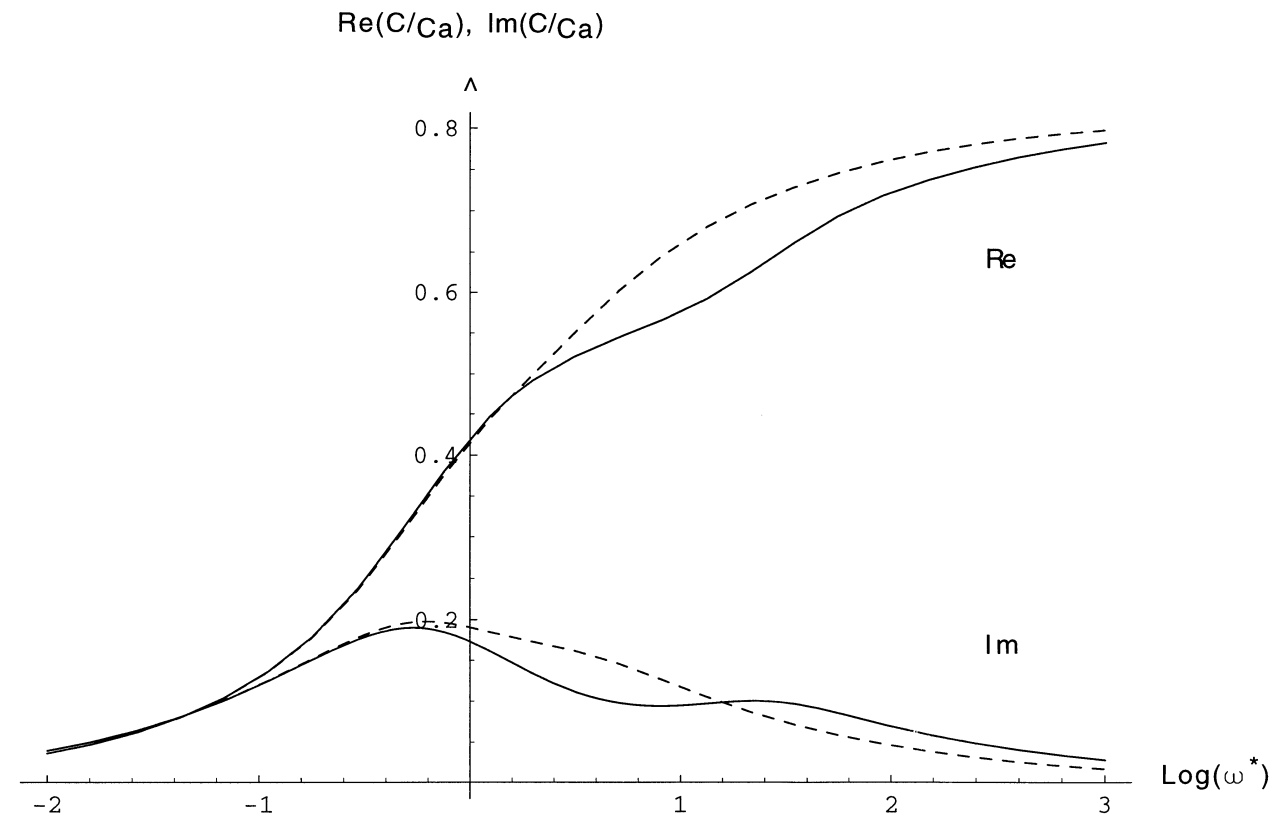

Fig. 5. Comparison between two distinct dual porosity media with the same pore structure but with different permeabilities in the micropores. Real and imaginary parts of complex-valued celerities, with respect to the dimensionless pulsation $\omega^{*}$. Solid line : example 1 in the conclusion $\left(k_{m}=k / 400\right)$. Dashed line: example 2 in the conclusion $\left(k_{m}=k / 2500\right)$.

$$
\begin{aligned}
f_{d} & \approx 815 \mathrm{~Hz} \\
f_{t m} & \approx 3 \mathrm{kHz}
\end{aligned}
$$

Figure 5 shows the acoustic properties of both dual porosity media defined above. 
In conclusion, these results prove that the introduction of a microporosity could be used to develop new porous materials, with improved of absorption in a given range of frequencies.

\section{REFERENCES}

Allard, J. F. (1993) Propagation of Sound in Porous Media. Modelling Sound Absorbing Materials. Chapman and Hall, London.

Allard, J. F., Herzog, P., Lafarge, D. and Tamura, M. (1993) Recent topics concerning the acoustics of fibrous and porous materials. Applied Acoustics 39, 3-21.

Attenborough, K. (1983) Acoustical characteristics of rigid fibrous adsorbents and granular media. Journal of the Acoustic Society of America. 73(3), 785-799.

Auriault, J. L. (1980) Dynamic behaviour of a porous media saturated by a Newtonian fluid. International Journal of Engineering Science 18, 775-785.

Auriault, J. L. (1983) Effective macroscopic description for heat conduction in periodic composites. International Journal of Heat and Mass Transfer 26(6), 861-869.

Auriault, J. L. and Boutin, C. (1993-1994) Deformable porous media with double porosity. I : Quasi-statics ; II : Memory effects; III : Acoustics. T.I.P.M. 7, 63-82; 10, 153-169; 14, 143-162.

Auriault, J. L. and Royer, P. (1993) Ecoulement d'un gaz dans un milieu à double porosité. Compte Rendu à l'Académie des Sciences, Paris 317(II), 431-436.

Auriault, J. L., Borne, L. and Chambon, R. (1985) Dynamic of porous saturated media. Checking of the generalized law of Darcy. Journal of the Acoustic Society of America 77, 1641-1650.

Bar, P. and Delanne, Y. (1993) Réduire le bruit Pneumatique-Chaussées. LCPC, Presses des Ponts et Chaussées.

Barenblatt, G. I., Entov, V. M. and Ryzhik, V. M. (1960) On fundamental equations of flow of homogeneous liquids in naturally fractured rocks. Dokl. Akad. Nauk., USSR 132(3), 545-548 (in Russian).

Biot, M. A. (1956) The theory of propagation of elastic waves in a fluid saturated porous solid, I. Low frequency range, II. Higher frequency range. Journal of the Acoustic Society of America 28, 168-191.

Boutin, C. (1994) Comportement macroscopique de matériaux hétérogeǹes. Thése d'Habilitation, Université Joseph Fourier, Grenoble.

Boutin, C. and Auriault, J. L. (1990) Dynamic behaviour of porous media saturated by a viscoelastic fluid. Application to bituminous concrete. International Journal of Engineering Science 28(11), 1157-1181.

Boutin, C. and Auriault, J. L. (1993) Acoustic of Newtonian fluid at large bubble concentration. Eur. J. Mech. B/Fluid 12(3), 367-399.

Boutin, C., Royer, P. and Auriault, J. L. (1996) Sound absorption of dry porous media with single and double porosity. 11th Conf. Eng. Mech. Div./ASCE., May 1996, Fort Lauderdale, FL., pp. 796-799.

Champoux, Y. and Allard, J. F. (1991) Dynamic tortuosity and bulk modulus in air saturated porous media. Journal of Applied Physics 70, 1975-1979.

Levy, T. (1979) Propagation of waves in a fluid saturated porous elastic solid. International Journal of Engineering Science 17, 105-114.

Sheng, P. and Zhou, M.-Y. (1988) Dynamic permeability in porous media. Physical Review Letters 61(41), 15911594.

Royer, P. (1994) Contribution de l'homogénéisation à l'étude de la filtration d'un gaz dans un milieu déformable à double porosite - application à l'étude du système gaz-charbon. Thèse de Doctorat, Université Joseph Fourier, Grenoble.

Royer, P. and Auriault, J. L. (1994) Transient quasi-static flow through a porous rigid medium with double porosity. T.I.P.M. 17(33), 33-57.

Royer, P., Auriault, J. L. and Boutin, C. (1996) Macroscopic modeling of double-porosity reservoirs. J. Pet. Sci. En. 16, 187-202.

Sanchez-Palencia, E. (1980) Non-homogeneous Media and Vibration Theory. Lecture Note in Physics, 127. SpringerVerlag, Berlin. 